\title{
Enhancement Technique of Image Contrast using New Histogram Transformation
}

\author{
Wanhyun $\mathrm{Cho}^{1}$, Seongchae $\mathrm{Seo}^{2}$, Jinho You ${ }^{3}$, Soonja Kang ${ }^{4}$ \\ ${ }^{1}$ Department of Statistics, Chonnam National University, Gwang-ju, Korea; ${ }^{2}$ Department School of Electronics \& Computer Engi- \\ neering, Chonnam National University, Gwang-ju, Korea; ${ }^{3}$ Doul Infotech, Gwang-ju, Korea; ${ }^{4}$ Department of Mathematical Educa- \\ tion, Chonnam National University, Gwang-ju, Korea. \\ Email: whcho@chonnam.ac.kr; scseo@chonnam.ac.kr; loafers@daum.net; sjkang@chonnam.ac.kr
}

Received November 2013

\section{ABSTRACT}

This paper presents a preprocessing technique that can provide the improved quality of image robust to illumination changes. First, in order to enhance the image contrast, we proposed new adaptive histogram transformation combining histogram equalization and histogram specification. Here, by examining the characteristic of histogram distribution shape, we determine the appropriate target distribution. Next, applying the histogram equalization with an image histogram, we have obtained the uniform distribution of pixel values, and then we have again carried out the histogram transformation using an inverse of target distribution function. Finally we have conducted various experiments that can enhance the quality of image by applying our method with various standard images. The experimental results show that the proposed method can achieve moderately good image enhancement results.

\section{KEYWORDS}

Image Preprocessing Technique; Contrast Enhancement; Histogram Equalization and Specification; Target Distribution Function

\section{Introduction}

Image enhancement is the preprocessing of image to improve the interpretability or perception of information in images for human viewers and to provide a better input for other automated image processing techniques. The principal objective of image enhancement is to modify attributes of an image to make it more suitable for a given task and a specific observer. During this process, one or more attributes of an image are modified. The choice of attributes and the way that they are modified are specific to a given task. Moreover, observer-specific factors, such as the human visual system and the observer's experience, will introduce a great deal of subjectively into the choice of image enhancement methods [1,2].

Image enhancement is generally used in the following three cases: noise reduction from image, contrast enhancement of the very dark and bright image, and highlight the edges of the objects in a blurring image. Noise reduction is the process of removing noise form a signal or an image. In general, images taken with both digital camera and conventional film cameras will pick up noise from a variety of sources. Therefore, it is required that the noise is removed for many further uses of these images. Contrast enhancement is acquiring clear image through brightness intensity value redistribution. That is, this is enhancing features as stretching interval between dark and brightness area. De-blurring process is to restore the sharp images via image de-convolution such as Wiener de-convolution.

Here, we are mainly interested in the contrast enhancement methods. Several methods have been proposed to achieve contrast enhancement invariant to illumination variations [3-7]. There are called as Gray level transformations, Gamma correction, Contrast stretching, Histogram equalization, Histogram matching and so on. A very popular one among contrast enhancement techniques is histogram equalization. This technique is performed by remapping the gray levels of the image based on the probability distribution of the input gray image levels. It flattens and stretches the dynamic range of the image's histogram and resulting in overall contrast enhancement. But, in spite of its popularity and simplicity, histogram equalization is not very suitable to be imple- 
mented in consumer electronics, such as digital TV, because the method tends to produce undesirable artifacts. Hence, many improved methods have been proposed to overcome these drawbacks. They are brightness preserving bi-histogram equalization (BBHE), dualistic subimage histogram equalization (DSIHE), minimum mean brightness error bi-histogram equalization (MMBEBHE), recursive mean-separate histogram equalization (RMSHE), recursive sub-image histogram equalization (RSIHE), multi-peak histogram equalization with brightness preserving (MPHEBP), and dynamic histogram equalization (DHE). On the other hand, another popular contrast enhancement scheme is histogram specification. This technique enables us to match the histogram of input image close to the histogram of target image. However, specifying the output histogram is not a smooth task as it varies from image to image. Hence, several researches have been proposed on improvement of histogram specification. They are dynamic histogram specification (DHS), Histogram specification with Gamma distribution (HSGD), image fusion histogram specification (IFHS), and automatic exact histogram specification (AEHS).

However, there are some problems when we have used the histogram equalization (HE) to improve the contrast of the image. First, the HE method does not take the mean brightness of an image into account. Second, the HE method may result in over enhancement and saturation artifacts due to the stretching of the gray levels over the full gray level range. Third, the HE method always yields the middle gray level regardless of the input image, and cause undesirable artifacts. Therefore, in order to improve these problems, we have decided in advance the target images and then we use the histogram specification method to an input image to get an image similar with target image. But, it is difficult that we determine in advance the appropriate target image. Hence, it is required to a new contrast enhancement method robust to illumination changes.

Hence, in order to solve these problems at the same time, we try to propose a new image enhancement method that uses a new histogram transformation. In the Section 2, in order to enhance the image contrast, we have considered new adaptive histogram transformation combining histogram equalization and histogram specification. In the Section 3, we presented experimental results that is able to demonstrate the effectiveness of the proposed method in comparison to a few existing methods quantitatively. And in the Section 4, we mentioned the conclusion of my paper.

\section{Contrast Enhancement}

\subsection{CDF Transformation}

Here we will propose a technique that can improve the contrast of an image by using a combination of Histo- gram Equalization (HE) and Histogram Specification (HS). This technique can be thought as the transformation that converts the values in the ranges between 0 and 1 given by histogram equalization of input image into pixel values of particular output image by using the specified cumulative distribution function (CDF) to achieve a well illuminated image. In our case, we adaptively select the CDF so that the contrast of image can automatically achieve an optimal level.

First, we assume to have an input image $\mathrm{I}(\mathrm{x}, \mathrm{y})$ with $\mathrm{N}$ pixels and a total number of $\mathrm{L}$ gray levels, e.g., 256 gray levels for an 8-bit image. We transform the distribution of the pixel intensity values in the image $\mathrm{I}(\mathrm{x}, \mathrm{y})$ into a uniform distribution on interval $[0,1]$ by using the histogram equalization defined as the following formula. For a grey level of $k, k=0,1, \cdots, L-1$, a new transformed one $\mathrm{u}_{\mathrm{k}}$ is defined by

$$
u_{k}=\sum_{i=0}^{k} \frac{n_{i}}{N}, k \in[0, \cdots, \mathrm{L}-1] .
$$

where $n_{i}$ denotes the number of pixels in $I(x, y)$ with the grey level value i. Equation (1) defines a mapping of the pixel' intensity values from their original range $[0, \cdots, \mathrm{L}-1]$ to the domain of $[0,1]$.

Second, if the distribution of desired target image $\mathrm{I}_{\mathrm{TA}}$ is specified, we define its probability density function (PDF) and CDF as follows:

$$
\mathrm{G}(\mathrm{z})=\int_{0}^{z} p_{\mathrm{z}}(\mathrm{v}) \mathrm{dv}, \mathrm{z} \in[0, \mathrm{~L}-1] .
$$

Third, we try to find a new value $\mathrm{k}_{\text {new }}$ corresponding with $\mathrm{u}_{\mathrm{k}}$ such that

$$
\mathrm{u}_{\mathrm{k}}=\mathrm{G}\left(\mathrm{k}_{\text {new }}\right)=\int_{0}^{k_{\text {new }}} p_{\mathrm{z}}(\mathrm{v}) \mathrm{dv} .
$$

But, since a new value $\mathrm{k}_{\text {new }}$ is continuous value scaled on an interval [0, L-1], we should take a Gauss bracket integer $\left[\mathrm{k}_{\mathrm{new}}+0.5\right]$.

Here, the procedure that we have considered so far would be expressed as the following example picture. Figure 1 shows the density histogram of a given image, its equalized histogram \& specified CDF, and the density histogram of output image.

Furthermore, we consider the three types of density histogram structures in order to determine adaptively a proper CDF form in our CDF transformation.

\subsection{Skew Histogram to the Right}

When it is given a dark image like as the following example image in Figure 2, its density histogram has a form skewed to the right.

In this case, the average of the gray values of a given image is smaller than the average value $(\mathrm{N}+1) / 2$ of symmetrical distribution. Furthermore, in order to im- 


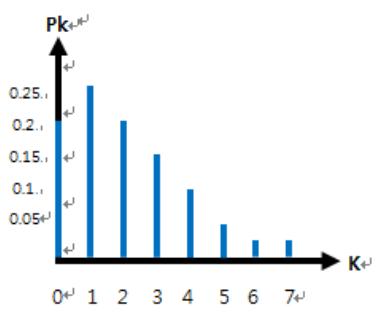

(a)

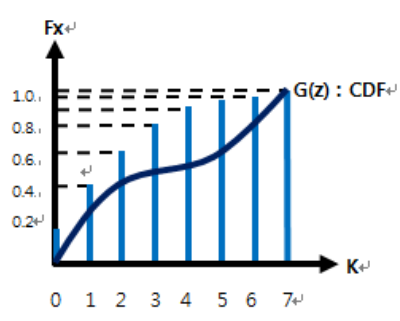

(b)

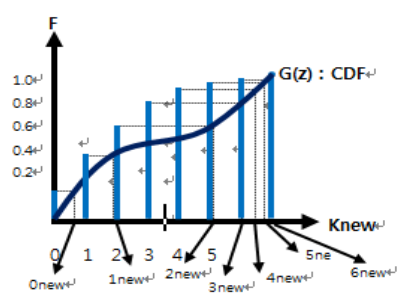

(c)

Figure 1. Graphical Representation of our procedure. (a) Histogram of synthetic image; (b) Its equalized histogram \& specified CDF; (c) Histogram transformation.
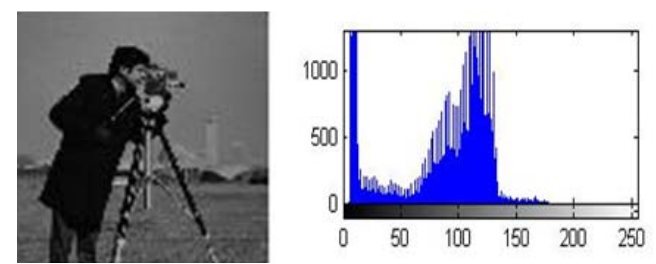

Figure 2. Dark sample image and its histogram.

prove the contrast of a given image, it is required with brighter image. Therefore, we have to consider a skew distribution to the left such as the Gompertz distribution with the opposite structure about a given histogram. Here, the expressions for its PDF, CDF and Inverse CDF are given respectively by

$$
\begin{aligned}
& p_{z}(z)=b \eta \exp ^{b z} \eta, \mathrm{b}\left(\theta \eta e^{b z}\right)> \\
& \mathrm{G}(\mathrm{z})=\mathrm{\eta} 1 \exp \left(-\left(e^{b z}-\right)\right)
\end{aligned}
$$

and $G^{-1}(\mathrm{u})=\frac{1}{b} \ln \left(1-\frac{1}{\eta} \ln (1-u)\right)$. Finally, when applying our method with CDF being Gompertz distribution to a given image, we have to select two parameters, $\eta$ and $b$. Here, we used $\eta=0.018$ and $b=2.322$. Figure 3 shows the contrast improved image and its histogram obtained by using our method.

\subsection{Skew Histogram to the Left}

As opposed to above, suppose that we have a very bright image like as the following example image in Figure 4. Then, its density histogram has a form skewed to the left.

In such a case, the average of the gray values of a giv-
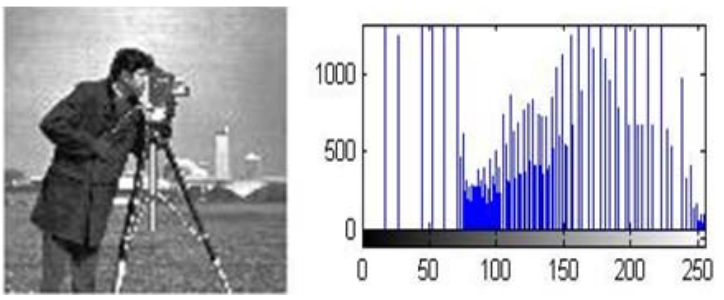

Figure 3. Contrast enhanced image and its histogram after applying CDF transformation.
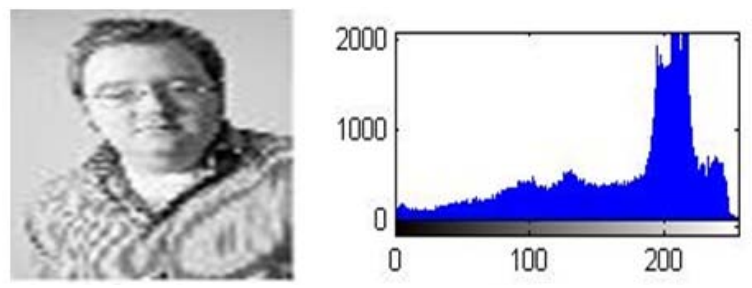

Figure 4. Bright sample image and its histogram.

en image is larger than the average value $(N+1) / 2$ of symmetrical distribution. Hence, in order to improve the contrast of a given image, it is required with darker image. Therefore, we have to consider a skew distribution to the right such as the log-normal distribution or the Weill distribution with the opposite structure about a given histogram. Here, the expressions for PDF, CDF, and inverse CDF of the Weibull distribution are given respectively by

$$
\begin{aligned}
& p_{z}(z)=\frac{k}{\lambda}\left(\frac{z}{}\right)^{k-1}, \mathrm{z} \geq \lambda 0>0, \mathrm{k}>0 \\
& \mathrm{G}(\mathrm{z})=1-\exp \left(-\left(\frac{\mathrm{z}}{\lambda}\right)^{k-1}\right)
\end{aligned}
$$

and $G^{-1}(u)=\lambda(1-1(-u))^{1 / k}$. Finally, when applying our method with CDF being Weibull distribution to a given image, we have to select two parameters, $\lambda$ (scale) and $\mathrm{k}$ (shape). Here, we used $\lambda=1.0$ and $\mathrm{k}=1.5$. Figure 5 shows the contrast improved image and its histogram obtained by using our method.

\subsection{Symmetrical Histogram}

Third, suppose that we have a usual image or equalized image like as the following example image in Figure 6. Its density histogram is generally given as the shape similar to the histogram of a symmetric distribution.

In this case, the average of the gray values of a given image is approximately equal to the average value ( $\mathrm{N}+$ 1)/2 of symmetrical distribution. Hence, in order to improve the contrast of a given image we have to consider a symmetric distribution such as the Normal distribution, the Student $\mathrm{t}$ distribution or the Logistic distribution. Here, we used the Normal distribution and its PDF and CDF are given respectively by 

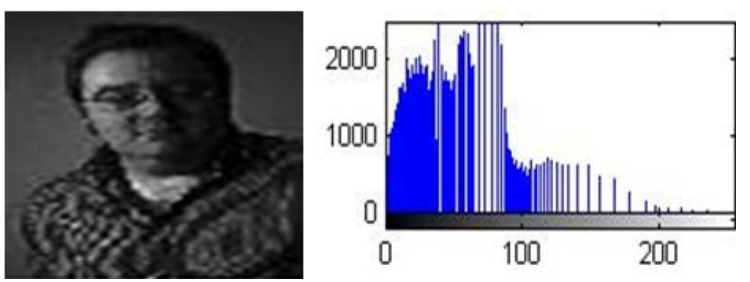

Figure 5. Contrast enhanced image and its histogram after applying CDF transformation.
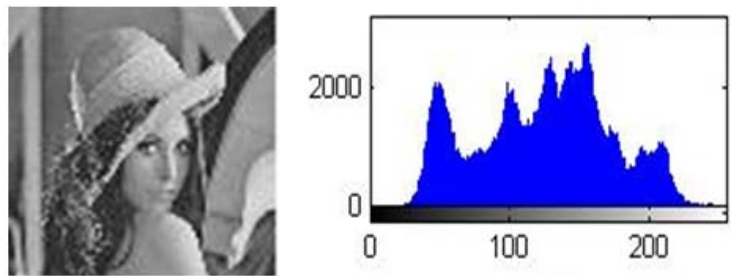

Figure 6. Usual sample image and its histogram.

$$
p_{z}(z)=\frac{1}{\sqrt{2 \pi} \sigma} \exp \left(-\frac{1}{2}\left(\frac{z-\mu}{\sigma}\right)^{2}\right),-\infty<\mu<\infty, \sigma>0
$$

and

$$
\mathrm{G}(\mathrm{z})=\int_{0}^{z} \frac{1}{\sqrt{2 \pi} \sigma} \exp \left(-\frac{1}{2}\left(\frac{u-\mu}{\sigma}\right)^{2}\right) d u
$$

Finally, when applying our method to a given image with CDF being the Normal distribution, we have to select two parameters, $\mu$ (location) and $\sigma$ (scale). Here, we used $\mu=127.5$ and $\sigma=14$. Figure 7 shows the contrast improved image and its histogram obtained by using our method.

\subsection{Adaptive Histogram Transformation}

Finally, we adaptively improve the contrast of input image by applying the following procedure. This method is summarized below.

1) Calculate the average for the gray values of the input image.

2) Determine whether this value belongs something among the three regions $[0, \mu-\sigma],[\mu-\sigma, \mu+\sigma]$ and $[\mu+\sigma$, 255], where $\mu$ and $\sigma$ are selected by the mean and the standard deviation of normal distribution. Here, we used $\mu=127.5$ and $\sigma=42$.

3) If this value belongs to the first region, we apply the Gomertz CDF method for input image, else if this value belongs to the second region, we apply the normal CDF method for input image, and else if this value belongs to the third region, we apply the Gumbel CDF method for input image.

\section{Experiments}

In this section, we provide experimental results in order to demonstrate the effectiveness of the proposed method in comparison to a few existing methods quantitatively. Here, we consider the performance of the proposed histogram transformation technique for global contrast enhancement of grayscale images. The performance of the various contrast enhancement methods were tested on standard images Camera man, Man, Barbara, Lena, Elaine. All of images are with size of $512 \times 512$ pixels. To compare their performances, the same images are enhanced with Gamma correction (GC), Histogram equalization (HE), Histogram specification (HS), and proposed method (PM). For all these methods, the performance is measure qualitatively in terms of human visual perception and quantitatively by using the widely used metric EME (a measure of enhancement) for measuring contrast enhancement. This measure is defined as follows:

$$
\mathrm{EME}=\frac{1}{k_{1} k_{2}} \sum_{l=1}^{k_{2}} \sum_{k=1}^{k_{1}} 20 \log \frac{I_{\text {max;k,l }}^{w}}{I_{m i n ; k, l}^{w}}
$$

where an image $\mathrm{I}(\mathrm{x}, \mathrm{y})$ is split into $\mathrm{k}_{1} \mathrm{k}_{2}$ blocks $\mathrm{W}_{\mathrm{k}, \mathrm{l}}(\mathrm{x}, \mathrm{y})$ of sizes $l_{1} \times l_{2}$, and $I_{\text {max; } k, l}^{w}$ and $I_{\text {min; }, l}^{w}$ are respectively maximum and minimum of the image $I(x, y)$ inside the block $\mathrm{W}_{\mathrm{k}, \mathrm{l}}(\mathrm{x}, \mathrm{y})$. The EME values for different images are given in Table 1. Here, Org, GC, HE, HS, and PM denote respectively for original image, gamma correction, histogram equalization, histogram specification, and propose method.

The enhanced images and histograms obtained by different methods for the Elaine image are shown in Figure 8.

It is evident from the table and figure that the proposed method provides better contrast enhancement than the existing methods.
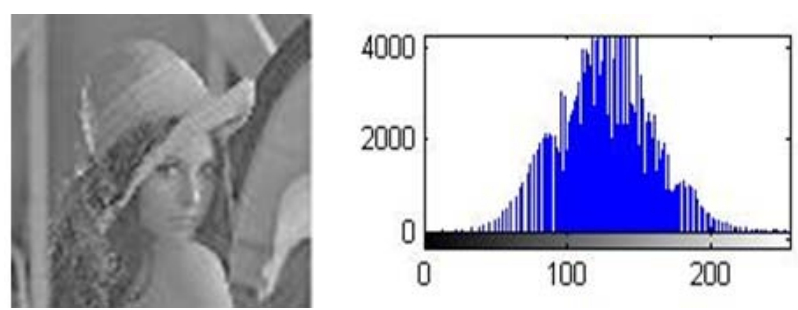

Figure 7. Contrast enhanced image and its histogram after applying CDF transformation.

Table 1. EME values for different methods and images.

\begin{tabular}{cccccc}
\hline & Cameraman & Man & Barbara & Lenna & Elaine \\
\hline Org & 16.962 & 10.741 & 11.966 & 18.030 & 14.303 \\
GC & 19.548 & 19.083 & 25.106 & 18.241 & 16.198 \\
HE & 12.757 & 20.956 & 18.864 & 18.764 & 17.207 \\
HS & 8.626 & 26.035 & 12.870 & 19.178 & 18.229 \\
PM & $\mathbf{8 . 0 7 5}$ & $\mathbf{2 3 . 8 8 6}$ & $\mathbf{1 5 . 6 8 8}$ & $\mathbf{1 8 . 6 6 6}$ & $\mathbf{1 8 . 3 3 7}$ \\
\hline
\end{tabular}




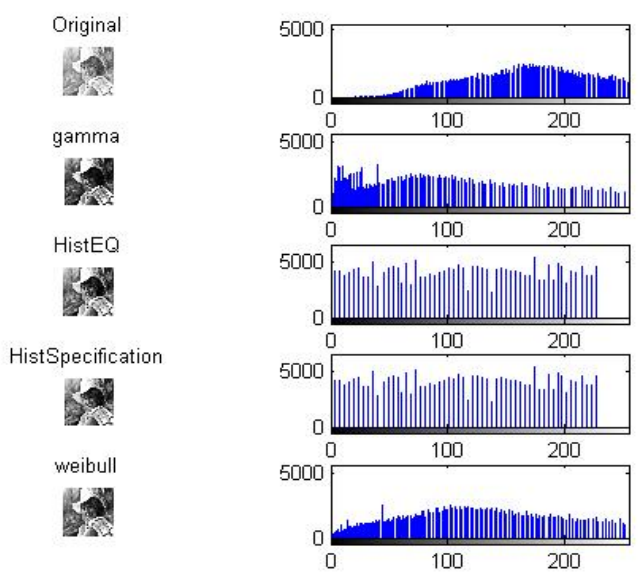

Figure 8. Enhanced images and histograms given by different methods.

\section{Conclusion}

In this paper, we propose a new image enhancement method that combines both the histogram transformation and edge-preserving regularization. First, we proposed new adaptive histogram transformation combining histogram equalization and histogram specification in order to enhance the image contrast. Second, we consider the characters of a various regularization functions in the energy functional that satisfies an edge-preserving noise reduction.

From the experimental results, we note that the proposed method can provide better contrast enhancement than the existing methods. And we note that the total variation method is considered as the best way to remove noise than other methods.

\section{Acknowledgements}

This work was supported in part by the Research Foundation Grant by the Chonnam National University (CNU-2012) and it also was the results of a study on the “Leades INdustry-university Cooperation” Project, sup- ported by the Ministry of Education, Science \& Technology (MEST).

\section{REFERENCES}

[1] R. Maini and H. Aggarwel, "A Comprehensive Review of Image Enhancement Techniques,” Journal of Computing, Vol. 2, No. 3, 2010, pp. 8-13.

[2] C. J. Prabhakar and P. U. Paraveen Kumar, “An Image Based Technique for Enhancement of Underwater Images,” International Journal of Machine Intelligence, Vol. 3, No. 4, 2011, pp. 217-224.

[3] H. Ibrahim and N. S. P. Kong, "Brightness Preserving Dynamic Histogram Equalization for Image Contrast Enhancement,” Consumer Electronics, Vol. 53, No. 4, 2007, pp. 1752-1758. http://dx.doi.org/10.1109/TCE.2007.4429280

[4] H. Yoon, Y. Han and H. Hahn, "Image Contrast Enhancement Based Sub-Histogram Equalization Technique without Over-Equalization Noise," International Journal of Electrical and Electronics Engineering, Vol. 3, No. 6, 2009, pp. 323-329.

[5] M. Kaur, J. Kaur and J. Kaur, "Survey of Contrast Enhancement Techniques Based on Histogram Equalization,” International Journal of Advanced Computer Science and Applications, Vol. 2, No. 7, 2011, pp. 137-141. http://dx.doi.org/10.14569/IJACSA.2011.020721

[6] V. Struc, J. Zibert and N. Pavesic, "Histogram Remapping as a Preprocessing Step for Robust Face Recognition," WSEAS Transactions on Information Science and Applications, Vol. 6, No. 3, 2009, pp. 520-529.

[7] K. K. Lavania and R. Shivali, Kumar, "A Comparative Study of Image Enhancement Using Histogram Approach," International Journal of Computer Applications, Vol. 32, No. 5, 2011, pp. 1-6.

[8] S. S. Agaian, K. P. Lentz and A. M. Grigoryan, "Transform-Based Image Enhancement Algorithms with Performance Measure," IEEE Transactions on Image Processing, Vol. 10, No. 3, 2001, pp. 367-381. http://dx.doi.org/10.1109/83.908502 